\title{
Exosomal Circ-XIAP Promotes Docetaxel Resistance in Prostate Cancer by Regulating miR-I I82/TPD52 Axis
}

\author{
Hui Zhang' \\ Minghui Li' \\ Jing Zhang' \\ Yanbing Shen ${ }^{2}$ \\ Qi Gui ${ }^{2}$ \\ 'College of Medical, Huanghuai \\ University, Zhumadian, Henan, People's \\ Republic of China; ${ }^{2}$ Department of \\ Urology, The Central Hospital of \\ Zhumadian, Zhumadian, Henan, People's \\ Republic of China
}

Background: Exosomal circular RNAs (circRNAs) are involved in the pathogenesis of prostate cancer $(\mathrm{PCa})$ and chemotherapy resistance. This research aimed to explore the function and molecular mechanism of circRNA X-linked inhibitor of apoptosis (circXIAP) in docetaxel (DTX) resistance of PCa.

Methods: The expression of circ-XIAP, microRNA-1182 (miR-1182), tumor protein D52 (TPD52) was measured by quantitative real-time polymerase chain reaction (qRT-PCR). Exosomes were detected with transmission electron microscopy (TEM). Cluster of differentiation 63 (CD63), cluster of differentiation 9 (CD9) and TPD52 protein levels were detected by Western blot (WB). FIfty percent inhibitory concentration (IC50) of DTX and cell viability were determined using Cell Counting Kit-8 (CCK-8) assay. Colony formation assay was applied to assess colony-forming ability. Cell cycle distribution and apoptosis were analyzed by flow cytometry. Transwell assay was used for measuring cell migration and invasion. Dual-reporter luciferase assay was performed to confirm the interaction between miR-1182 and circ-XIAP or TPD52. The role of circ-XIAP in vivo was confirmed via the mice xenograft model.

Results: Circ-XIAP and TPD52 were upregulated and miR-1182 was downregulated in DTX-resistant PCa tissue specimens and cell lines. Circ-XIAP was also overexpressed in exosomes from DTX-resistant cells and could be transmitted via exosomes. Circ-XIAP knockdown enhanced DTX sensitivity by suppressing DTX-resistant cell proliferation, migration and invasion and inducing cell cycle arrest and apoptosis. Circ-XIAP directly targeted miR-1182, and the effects of circ-XIAP knockdown were reversed by downregulating miR-1182 in DTX-resistant cells. TPD52 was the target of miR-1182, and its upregulation weakened the promotive effect of miR-1182 on DTX sensitivity. Importantly, circ-XIAP depletion inhibited tumor growth and increased DTX sensitivity in vivo.

Conclusion: Exosomal circ-XIAP promoted DTX resistance of PCa by regulating miR1182/TPD52 axis, providing a promising therapeutic target for PCa chemotherapy.

Keywords: prostate cancer, exosomes, circ-XIAP, miR-1182, TPD52

\section{Introduction}

Prostate cancer (PCa) is a common malignant tumor and is the fifth leading cause of cancer death in men (359,000 associated deaths in 2018). ${ }^{1}$ Although comprehensive treatments have been developed, the prognosis of PCa patients is still unsatisfactory because of the recurrence and metastasis. ${ }^{2}$ Docetaxel (DTX)-based chemotherapy is a first-line chemotherapy for the treatment of PCa that can prolong the survival of
College of Medical, Huanghuai University, No. 2628, Mountain Panlong Road,

Zhumdian, 463000, Henan, People's

Republic of China

Tel +86-0396-2853503

Email cecile98@I63.com 
patients. ${ }^{3}$ However, many PCa patients gradually develop resistance to DTX treatment during chemotherapy, resulting in tumor recurrence. ${ }^{4}$ Hence, understanding the underlying mechanism of DTX resistance is of great significance to improve the prognosis of $\mathrm{PCa}$ patients.

Exosomes are a class of extracellular vesicles with a size of $40-100 \mathrm{~nm}$, which originate from endosomal multivesicular bodies. ${ }^{5}$ Exosomes are secreted by most cells and contain complex and rich RNAs and proteins. ${ }^{6}$ Exosomes have been confirmed to be involved in regulating the tumor-normal communication in the tumor microenvironment. ${ }^{7}$ As key mediators of intercellular communication, exosomes can regulate the biological processes of cells and serve as crucial biomarkers for various diseases. ${ }^{8,9}$ Moreover, exosomes have been demonstrated to play an active role in tumor development (including PCa) and DTX resistance. ${ }^{10,11}$ Nevertheless, the components of exosomes secreted by different cells are complex and different, so more exosomal RNA molecules need to be discovered and explored.

CircRNAs, a special type of non-coding RNAs (ncRNAs), form continuous covalently closed-loops with neither 5 '-end cup nor $3^{\prime}$-end ploy A tail. ${ }^{12}$ Moreover, circRNA have strong stability due to their covalently closed continuous loop structures. ${ }^{13}$ Increasing evidence indicates that circRNAs participate in regulating many physiological and pathological processes in human cancers. ${ }^{14,15}$ Moreover, circRNAs have been confirmed to be involved in participate in DTX resistance in many cancers, including PCa. ${ }^{16,17}$ Previous researches have indicated that circRNAs are stable and enriched in exosomes. ${ }^{18}$ Moreover, it has been reported that exosomal circRNAs can be served as potential biomarkers and new therapeutic targets for diseases. ${ }^{19}$ CircRNA X-linked inhibitor of apoptosis (circ-XIAP) is derived from mRNA back-splicing of XIAP gene and located at chrX: 123022468-123026623, and it acts as an oncogene in PCa. ${ }^{20}$ Nevertheless, the functions and molecular mechanism of exosomal circ-XIAP in DTX resistance of PCa have not been reported.

CircRNAs can function as microRNAs (miRNAs) sponges by competitively binding to miRNA response elements, thus regulating the biological activity of miRNA and the functional role of miRNA on target genes. ${ }^{21} \mathrm{MiR}-1182$ has been shown to inhibit proliferation, metastasis and tumorigenesis in many cancers. $^{22,23}$ Recently, miR-1182 was found to function as a tumor suppressor in $\mathrm{PCa}^{24}$ However, whether circ-XIAP can modulate the resistance of PCa to DTX through interacting with miR-1182 is still unclear. Tumor protein D52 (TPD52), a chromosome 8q21 amplification target, acts as an oncogene in many cancers, including $\mathrm{PCa}^{25,26}$ Coincidently, by bioinformatics analysis, we found that both circ-XIAP and 3'UTR of TPD52 had the complementary binding sequence for miR-1182, which suggested the interactions among circ-XIAP, miR-1182 and TPD52 in DTX resistance of $\mathrm{PCa}$.

In our research, we aimed to investigate the biological functions of exosome-transmitted circ-XIAP in DTX resistance of $\mathrm{PCa}$, and search for the underlying mechanism mediated by circ-XIAP, hoping to find novel targets for the treatment of DTX-resistant PCa patients.

\section{Materials and Methods Patient Samples}

$\mathrm{PCa}$ tissue specimens ( $\mathrm{N}=52)$ were acquired from the $\mathrm{PCa}$ patients after surgical resection at The Central Hospital of Zhumadian. These PCa patients were divided into two groups based on their sensitivity to DTX: sensitive $(\mathrm{N}=23)$ and resistant $(\mathrm{N}=29)$. These tissues specimens were collected, timely frozen in liquid nitrogen and then kept in a refrigerator at $-80^{\circ} \mathrm{C}$ after surgical resection. The research has been carried out in accordance with the World Medical Association Declaration of Helsinki, These patients had provided informed consents. Our experiments were approved by the Research Ethics Committee of The Central Hospital of Zhumadian.

\section{Cell Culture and Transfection}

Four PCa cell lines (22Rv1, VCaP, DU145, and PC3) and human normal prostate epithelial cells (RWPE-1) were obtained from COBIOER (Nanjing, China). All cells were allowed to grow in RPMI 1640 medium (Invitrogen, Carlsbad, CA, USA) that contained 10\% FBS (Sigma-Aldrich, St. Louis, MO, USA) in a humidified air with $5 \% \mathrm{CO}_{2}$ at $37^{\circ} \mathrm{C}$. To establish DTXresistant PCa cells (DU145/DTX and PC3/DTX), DU145 and PC3 cells were exposed to increasing doses of DTX (Sigma-Aldrich). Then, DTX-resistant cells were treated with DTX $(5 \mathrm{nM})$ to maintain a resistant phenotype.

Small interference RNA against circ-XIAP (si-circXIAP) and matched negative control (si-NC), circ-XIAP or TPD52 overexpression vector (circ-XIAP or TPD52) and matched negative control (vector), miR-1182 mimics (miR-1182) and match negative control (miR-NC), miR- 
1182 inhibitor (anti-miR-1182) and match negative control (anti-miR-NC) were synthesized by GeneCopoeia (Guangzhou, China). Lentivirus packaged plasmid sh-circXIAP and its negative control (sh-NC) were provided by RiboBio (Guangzhou, China). Cells were transfected by using Lipofectamine 3000 (Invitrogen).

\section{RNA Isolation and Quantitative Real-Time Polymerase Chain Reaction (qRT-PCR)}

TRIzol reagent (Invitrogen) was applied for isolation of total RNA. Total exosomal RNAs were acquired using the exoRNeasy Midi Kit (Qiagen, Valencia, CA, USA). The Prime Script RT reagent Kit (TaKaRa, Kusatsu, Japan) or miRNA cDNA synthesis kit (Ribobio) was applied for reverse transcription. Then, qRT-PCR was performed on a CFX Realtime PCR system (Bio-Rad, Hercules, CA, USA) using SYBR Green PCR Kit (Takara). Gene expression was detected according to the 2- $\Delta \Delta \mathrm{Ct}$ method, followed by normalization to GAPDH (for circ-XIAP, XIAP and TPD52) or U6 (for miR1182). Primer sequences: circ-XIAP (F, 5'-CATTCACTTG AGGAGTGTCTGG-3'; R, 5'-TTGTTCCCAAGGGTCTTC AC-3'); XIAP (F, 5'-TGGCAGATTATGAAGCACGGATC -3'; R, 5'-AGTTAGCCCTCCTCCACAGTGA-3'); miR-1182 (F， 5'-GCGGAGGGTCTTGGGAGG-3'; R， 5'-CAGTG CGTGTCGTGGAGT-3'); TPD52 (F, 5'-AACAGAACATTG CCAAAGGGTG-3'; R, 5'-TGACTGAGCCAACAGACG AAA-3'). GAPDH (F, 5'-GTCTCCTCTGACTTCAACA GCG-3'; R, 5'-ACCACCCTGTTGCTGTAGCCAA-3'), U6 (F， 5'-CTCGCTTCGGCAGCACA-3'; R， 5'-AACGCTT CACGAATTTGCGT-3').

\section{RNase R Treatment}

To determine the circular characteristic of circ-XIAP, total RNA $(2 \mu \mathrm{g})$ was treated with or without RNase R (6 units, Geneseed Biotech, Guangzhou, China) for $0.5 \mathrm{~h}$ at $37^{\circ} \mathrm{C}$. Lastly, the enrichment of circ-XIAP and XIAP mRNA was tested by qRT-PCR analysis.

\section{Subcellular Fractionation Location}

The Cytoplasmic \& Nuclear RNA Purification Kit (Norgen Biotek, Thorold, Canada) was applied for isolating cytosolic and nuclear fractions. Afterwards, the expression was detected via qRT-PCR analysis in nuclear and cytosolic fractions. U6 or GAPD was used as a control for the nucleus and cytoplasm, respectively.

\section{Cell Counting Kit-8 (CCK-8) Assay}

CCK-8 purchased from Boster (Wuhan, China) was used for analyzing 50\% inhibitory concentration (IC50) value of DTX and cell viability. In brief, DU145/DTX and PC3/ DTX cell suspension $\left(100 \mu \mathrm{L}, 2 \times 10^{3}\right.$ cells $)$ was added into a 96 -well plate. CCK-8 reagent $(10 \mu \mathrm{L})$ was added to per well after respective treatment. After incubation for $2-3 \mathrm{~h}$, a microplate reader (Bio-Rad) was applied to examine the absorbance of per well at $450 \mathrm{~nm}$. $\mathrm{IC}_{50}$ value of DTX was calculated using GraphPad Prism.

\section{Exosome Isolation and Identification}

ExoQuick precipitation kit (System Biosciences, Mountain view, CA, USA) was utilized for isolating exosomes from cell culture medium following the manufacturer's instructions. In short, cell culture medium was collected and centrifuged $(3000 \times \mathrm{g}, 15 \mathrm{~min})$, followed by addition of ExoQuick precipitation solution to supernatant. After centrifugation $(1500 \times \mathrm{g}, 30 \mathrm{~min})$, exosome pellet was resuspended in phosphate-buffered saline (PBS; Beyotime, Shanghai, China). Exosomes were visualized by transmission electron microscopy (TEM; JEOL, Akishima, Japan) and exosome protein markers (CD63 and CD9) were identified by WB assay.

\section{Western Blot (WB) Assay}

RIPA lysis buffer (Beyotime) was applied for extracting the total protein. After measurement of protein concentration, protein (about $40 \mu \mathrm{g} / \mathrm{lane}$ ) was subjected to SDSPAGE (Beyotime). Subsequently, the proteins were transferred to nitrocellulose membranes (Invitrogen), which were then blocked with 5\% non-fat milk (Yili, Beijing, China) and incubated with primary antibody overnight at $4^{\circ} \mathrm{C}$. The primary antibodies including cluster of differentiation 63 (CD63; 1:1000, ab118307), cluster of differentiation 9 (CD9; 1:500, ab223052), TPD52 (1:5000, ab155296), and GAPDH (1:2000, ab37168) were bought from Abcam (Cambridge, UK). After that, the corresponding secondary antibody (1:4000, ab205718, Abcam) was used for the combination with the primary antibody. At last, the visualization of protein blots was achieved by an enhanced chemiluminescence reagent (Solarbio, Beijing, China).

\section{Colony Formation Assay}

Transfected cells (DU145/DTX and PC3/DTX) were plated into 12-well plates. The cells were then cultured for 14 
days, and the medium was replaced as needed. After that, the colonies were fixed with ethanol $(75 \%$, Beyotime), followed by staining with violet $(0.1 \%$, Beyotime $)$. At last, colonies ( $>50$ cells/colony) were counted and photographed.

\section{Flow Cytometry Analysis}

For analysis of cell cycle progression, DU145/DTX and PC3/DTX cells were collected, followed by fixing with ice-cold ethanol $\left(75 \%\right.$, Beyotime) at $-20^{\circ} \mathrm{C}$ for $12 \mathrm{~h}$. Next, the fixed cells were subsequently incubated with propidium iodide (PI; Sangon Biotech, Shanghai, China) and RNase A (Sangon Biotech) in the darkness for $0.5 \mathrm{~h}$. The cells were analyzed by flow cytometry (BD Biosciences, Franklin, NJ, USA) for determination of cell cycle distribution. The apoptosis assay was conducted with the Annexin V-FITC/PI apoptosis detection kit (Sangon Biotech) via suspending the cells in binding buffer ( 400 $\mu \mathrm{L}$ ) containing Annexin V-FITC and PI, incubating them in the darkness for $20 \mathrm{~min}$. Finally, flow cytometry was utilized to quantify cell apoptosis.

\section{Transwell Assay}

Transwell assay was performed by transwell inserts ( $8 \mu \mathrm{m}$ pore-size, ( $8 \mathrm{~mm}$ pores; Corning, Costar, NY, USA) either without Matrigel (for migration assay) or with Matrigel (for invasion assay) (BD Biosciences). Briefly, the serumfree media containing transfected cells was added to the top chamber, and the medium with $10 \%$ FBS was added to the lower part of the chamber. The cells on the bottom membrane surface were fixed in ethanol (Beyotime) and stained by crystal violet (Beyotime) after $24 \mathrm{~h}$ of incubation. Images were captured under a microscope (Leica, Wetzlar, Germany) at $\times 100$ magnification.

\section{Dual-Luciferase Reporter Assay}

The putative binding sequence of miR-1182 and circXIAP or TPD52 was predicted by Circinteractome or TargetScan. Wide-type (WT) sequence of circ-XIAP or TPD52 3'UTR with binding sequence for miR-1182 was cloned into the pmirGLO vector (YouBia, Changsha, China) to construct WT-circ-XIAP or WT-TPD52 3'UTR. At the same time, mutant reporter plasmids (MUT-circXIAP or MUT-TPD52 3'UTR) without binding sequence for miR-1182 were generated in the same way. Then, the indicated vector and miR-NC/miR-1182 were cotransfected into DU145/DTX and PC3/DTX cells. After $48 \mathrm{~h}$ of co-transfection, dual-luciferase reporter assay system (Promega, Madison, WI, USA) was applied for examining the luciferase activity.

\section{Immunohistochemistry Analysis}

Human tumor tissues from sensitive and resistant tissues were fixed in formalin solution (Beyotime) and dehydrated in ethanol, embedded in paraffin, and cut. Next, consecutive thick sections $(4 \mu \mathrm{m})$ were incubated with antibodies against TPD52 (1:5000, ab155296) for $12 \mathrm{~h}$ at $4^{\circ} \mathrm{C}$. After that, the sections were incubated with secondary antibody (1:5000, ab205718, Abcam) for $1 \mathrm{~h}$. The sections were stained by diaminobenzidine (DAB) complex, and the nuclei were counterstained with haematoxylin. At last, the sections were examined with a fluorescence microscope (Leica).

\section{In vivo Tumor Model}

BALB/c nude mice ( $\mathrm{N}=32$, male, 5 weeks, weighing $18 \pm$ $2 \mathrm{~g})$ used in this study were bought from Laboratory Animal, Inc., (Beijing, China). PC3/DTX cells $\left(3 \times 10^{6} \%\right.$ $0.2 \mathrm{~mL}$ PBS, circ-XIAP silencing or control) were subcutaneously injected into nude mice. The circ-XIAP silencing or control group was randomly divided into two groups ( $\mathrm{N}=8 /$ group). After injection for 7 days, tumor volume was measured and one group was intraperitoneally injected with DTX $(10 \mathrm{mg} / \mathrm{kg})$ every 3 days. Tumors volume was calculated based on the formula: volume=length $\times$ width $^{2} \times 0.5$. After 22 days, these mice were killed by cervical dislocation following exposure to $\mathrm{CO}_{2}$ (30\% of the chamber volume was displace per minute by the flow of $\mathrm{CO}_{2}$ ), and tumor tissues were photographed, weighed and harvested for detecting the expression levels of circ-XIAP, miR-1182 and TPD52. The in vivo experiments obtained the approval from the Animal Care and Use Committee of The Central Hospital of Zhumadian. Animal studies were performed in compliance with the ARRIVE guidelines and the Basel Declaration. All animals received humane care according to the National Institutes of Health (USA) guidelines.

\section{Statistical Analysis}

Experimental data from at least three independent experiments were displayed as mean \pm standard deviation (SD) and analyzed by GraphPad Prism. Student's $t$-test or a one-way ANOVA was used for assessing significance of differences between two groups or among multiple groups. Pearson correlation coefficient was applied for analyzing the correlation between miR-1182 and circ-XIAP or TPD52. $P$ value $<0.05$ was considered to be statistically significant. 


\section{Results}

\section{Circ-XIAP Was Upregulated in PCa Cells} and DTX-Resistant PCa Tissues and Cells

To explore whether circ-XIAP was dysregulated in $\mathrm{PCa}$ cells, its expression was detected by qRT-PCR. Compared to RWPE-1 cells, the expression of circ-XIAP was increased in PCa cells (22Rv1, VCaP, DU145, and PC3), especially in DU145 and PC3 cells (Figure 1A). Next, we chose DU145 and PC3 cells for further study. In contrast to the linear XIAP mRNA, circ-XIAP was resistant to RNase R (Figure $1 \mathrm{~B}$ and $\mathrm{C}$ ), indicating that circ-XIAP had a loop structure. Moreover, circ-XIAP was primarily located in the cytoplasm (Figure 1D and E). PCa tissues were divided into 2 groups: sensitive $(\mathrm{N}=23)$ group and resistant $(\mathrm{N}=29)$ group, according to the patient outcomes after DTX treatment. The data indicated that circ-XIAP level was enhanced in DTXresistant tissue samples compared to DTX-sensitive tissue samples (Figure 1F). In addition, circ-XIAP was also upregulated in tumor tissues compared with the adjacent normal tissues (Supplementary Figure 1). Moreover, $\mathrm{IC}_{50}$ of DTX was significantly increased in DTX-resistant cells (DU145/ DTX and PC3/DTX) cells relative to parental cells (Figure $1 \mathrm{G})$, indicating that resistant cells respond less to DTX treatment. In addition, the level of circ-XIAP was higher in DTX-resistant cells than parental cells (Figure 1H). Collectively, circ-XIAP might play a vital role in DTX resistance.

\section{Circ-XIAP Could Be Delivered Through Exosomes}

Exosomes act as crucial mediators of intercellular communication. Next, exosomes in DTX-resistant cell culture media were isolated. As shown in Figure 2A, exosomes of DU145/DTX and PC3/DTX cells were observed under a TEM. WB was conducted for detecting the levels of exosome protein markers (CD63 and CD9). Results showed that CD63 and CD9 were all highly enriched in cell exosomes, but not in supernatant (Figure 2B). Moreover, the expression of circ-XIAP in exosomes (exocirc-XIAP) was measured. As presented in Figure 2C, exocirc-XIAP expression in DU145 and PC3 cells was markedly higher than in RWPE-1 cells and strikingly lower than in DU145/DTX and PC3/DTX cells. Subsequently, we further explored whether circ-XIAP was secreted by incorporation into exosomes. Treatment of exosomes from DTX-resistant cells increased circ-XIAP expression in DU145 and PC3 cells (Figure 2D), suggesting that circXIAP-contained exosomes could be absorbed by recipient cells. GW4869 has been successfully used to block the secretion of exosomes from cells. As shown in Figure 2E, GW4869 treatment decreased circ-XIAP expression in DU145/DTX and PC3/DTX cells, indicating that reduction of exosomes would reduce the expression of circ-XIAP. These data illustrated that circ-XIAP was secreted via incorporation into exosomes in cells.
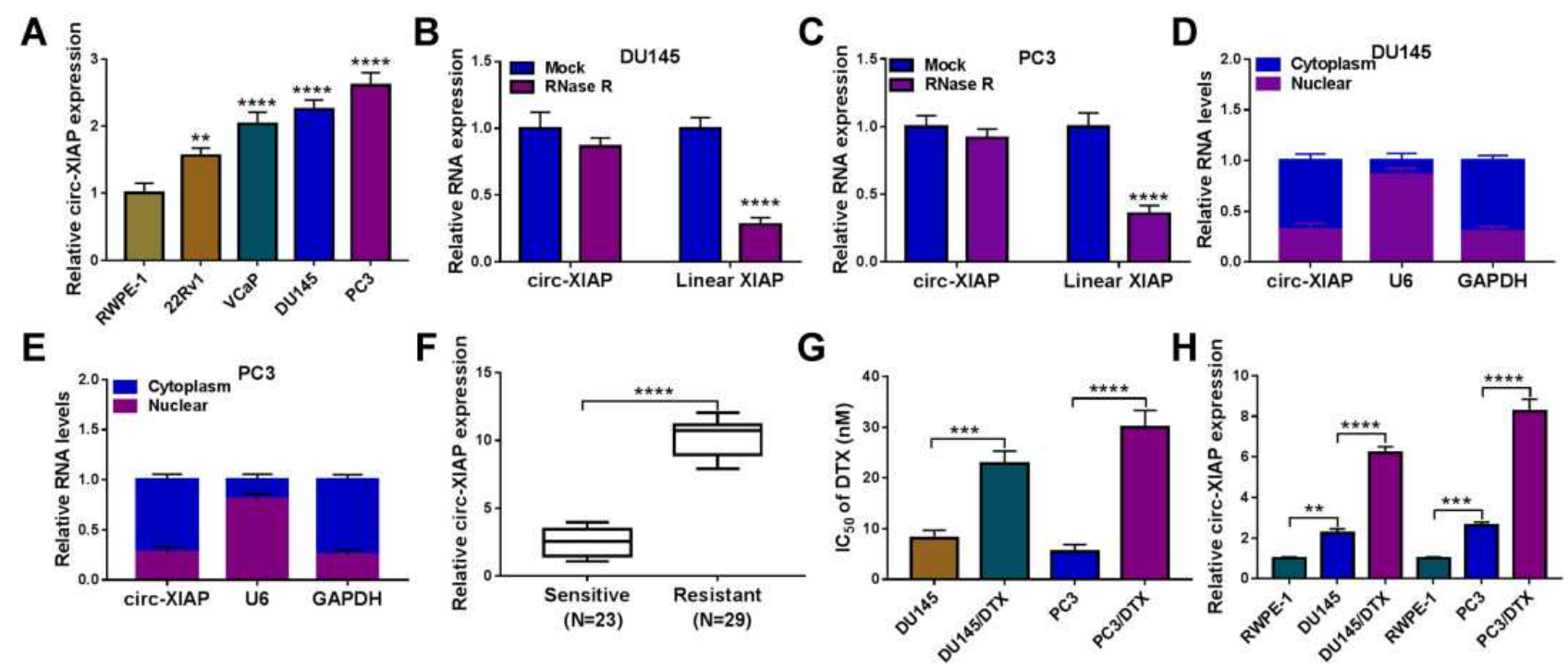

Figure I Relative expression of circ-XIAP in PCa cells and DTX-resistant PCa tissue samples and cells. (A) Circ-XIAP expression was detected via qRT-PCR in RWPE-I and PCa cells (22RvI, VCaP, DUI45, and PC3). (B and C) After treatment with RNase R, circ-XIAP and linear XIAP mRNA expression were measured in DUI45 and PC3 cells. (D and E) The subcellular location of circ-XIAP in DUI45 and PC3 cells was analyzed by qRT-PCR. (F) Circ-XIAP expression was examined in DTX-sensitive (N=23) and DTX-resistant $(\mathrm{N}=29)$ tissues. $(\mathrm{G}) \mathrm{IC}_{50}$ value of DTX was determined using CCK-8 analysis in DUI45, DUI45/DTX, PC3, and PC3/DTX cells. (H) The level of circ-XIAP was measured in RWPE-I, DUI45, DUI45/DTX, PC3, and PC3/DTX cells. $* * P<0.01$, $* * * P<0.001$, $* * * * P<0.0001$. 
A

DU145/DTX
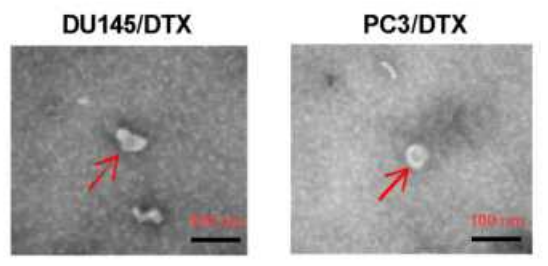

D

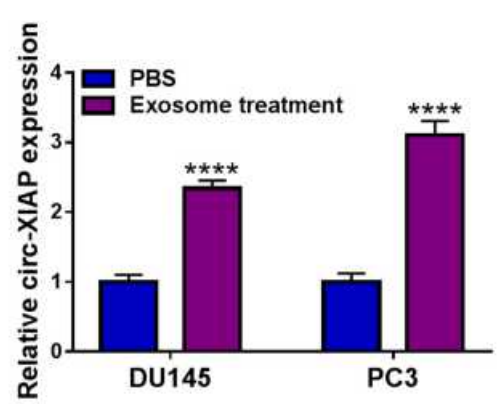

B

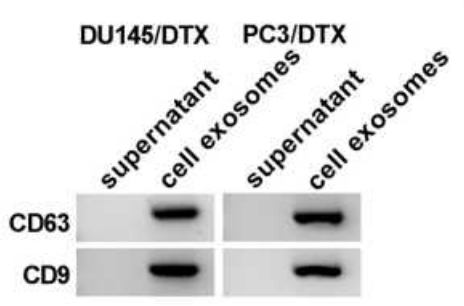

E

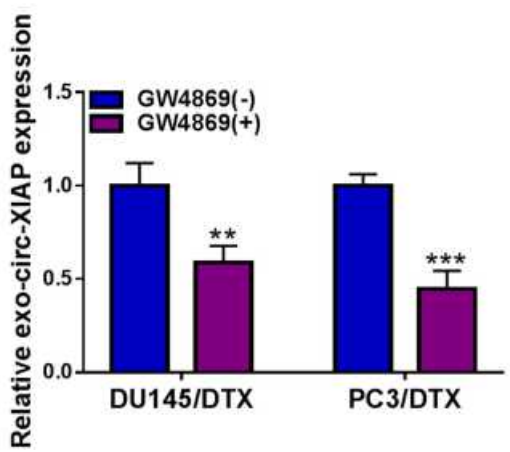

Figure 2 Circ-XIAP could be transferred via exosomes. (A) Exosomes secreted by DUI45/DTX and PC3/DTX cells were observed by TEM. (B) WB assay was conducted for determination of CD63 and CD9 protein levels in the supernatant or exosomes of DUI45/DTX and PC3/DTX cells. (C) Circ-XIAP expression in exosomes of RWPE-I, DUI45, DUI45/DTX, PC3, and PC3/DTX cells was examined. (D) Circ-XIAP expression in DUI45 and PC3 cells treated with PBS or exosome was detected. (E) Circ-XIAP expression was measured in DUI45/DTX and PC3/DTX cells treated with or without GW4869. $* * P<0.01, * * * P<0.001$, $* * * * P<0.0001$.

Knockdown of Circ-XIAP Enhanced DTX Sensitivity in DTX-Resistant PCa Cells

First, knockdown and overexpression efficiency of circXIAP were verified via qRT-PCR (Figure 3A). Since upregulation of circ-XIAP contributed to DTX resistance, we next examined whether knockdown of circXIAP would re-sensitize these cells to DTX treatment. Toward this purpose, we silenced circ-XIAP expression by transfection of si-circ-XIAP. We found that $\mathrm{IC}_{50}$ value of DTX was decreased in after knockdown of circ-XIAP (Figure 3B). Moreover, interference of circXIAP led to significant inhibition of cell viability and colony formation rate (Figure $3 \mathrm{C}$ and $\mathrm{D}$ ), suggesting that circ-XIAP knockdown inhibited cell proliferation. In addition, flow cytometry results manifested that more cells were distributed in the G0/G1 phase, and fewer cells were distributed in $\mathrm{S}$ phase after downregulating circ-XIAP (Figure 3E), implying that cell cycle was arrested in G0/G1 phase. Furthermore, circ-XIAP downregulation exhibited significantly higher apoptosis rate (Figure 3F). Meanwhile, DU145/DTX and PC3/DTX cell migration and invasion were inhibited by circ-
XIAP silence (Figure 3G and H). Next, we explored the role of circ-XIAP upregulation in parental $\mathrm{PCa}$ cells. Overexpression efficiency of circ-XIAP was shown in Supplementary Figure 2A. Overexpression of circ-XIAP increased $\mathrm{IC}_{50}$ value of DTX, cell viability, colony formation ability, migration, and invasion in DU145 and PC3 cells (Supplementary Figure 2B-2F). Together, these data implicated that circ-XIAP downregulation could re-sensitize DU145/DTX and PC3/ DTX cells to DTX.

\section{Circ-XIAP Was a Sponge of miR-II 82}

CircRNAs can exert their function as miRNA sponges. ${ }^{27}$ Next, we used online bioinformatics database (Circinteractome and circbank) to predict the potential targets of circ-XIAP. We found that two miRNAs (miR-507 and miR-1182) were overlapped in Circinteractome and Circbank (Supplementary Figure 3A). Moreover, we found that miR-507 and miR-1182 expression were increased by transfection of si-circXIAP, and the expression of miR-1182 was higher than that of miR-507 expression (Supplementary Figure 3B). Therefore, miR-1182 was selected for 

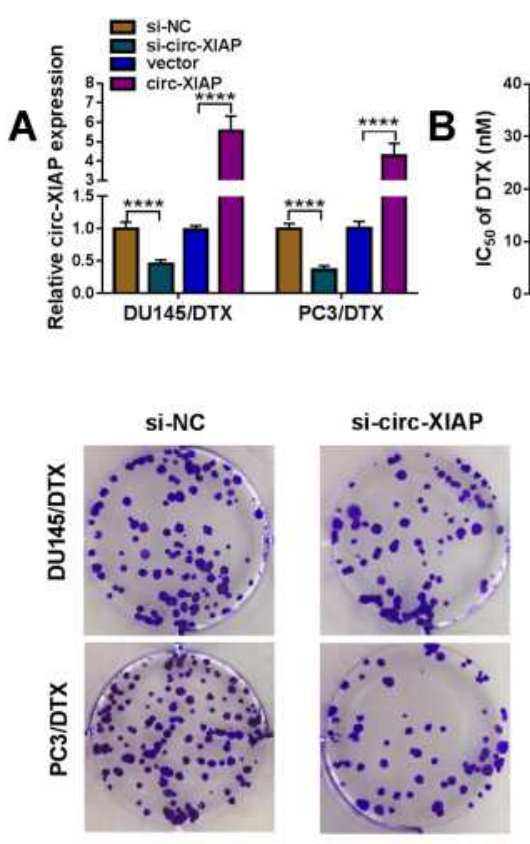

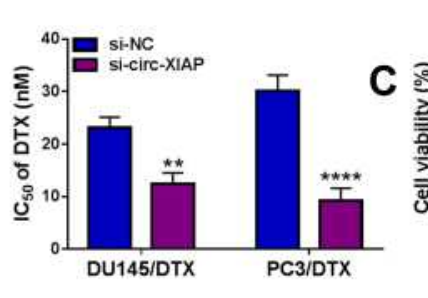

E
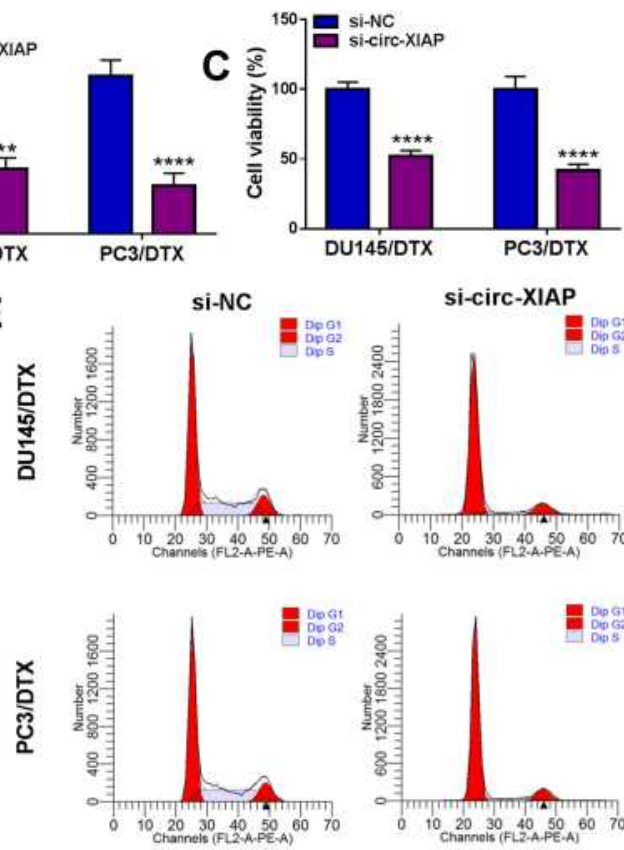

D
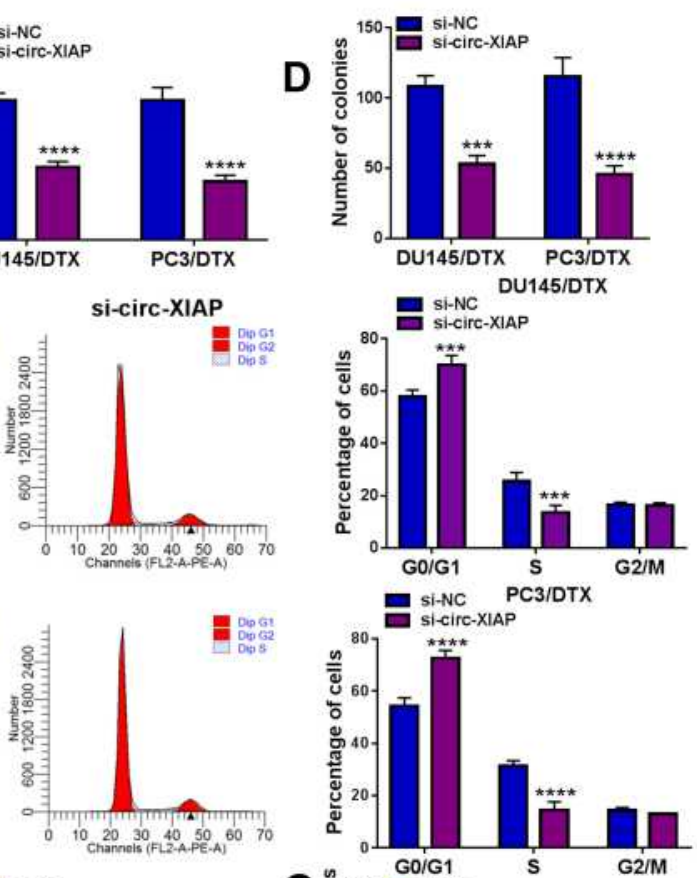

$\mathbf{F}$
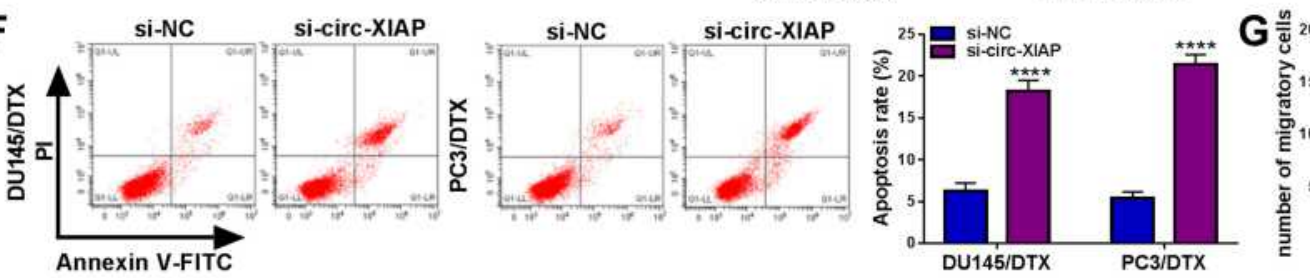

G0/G1
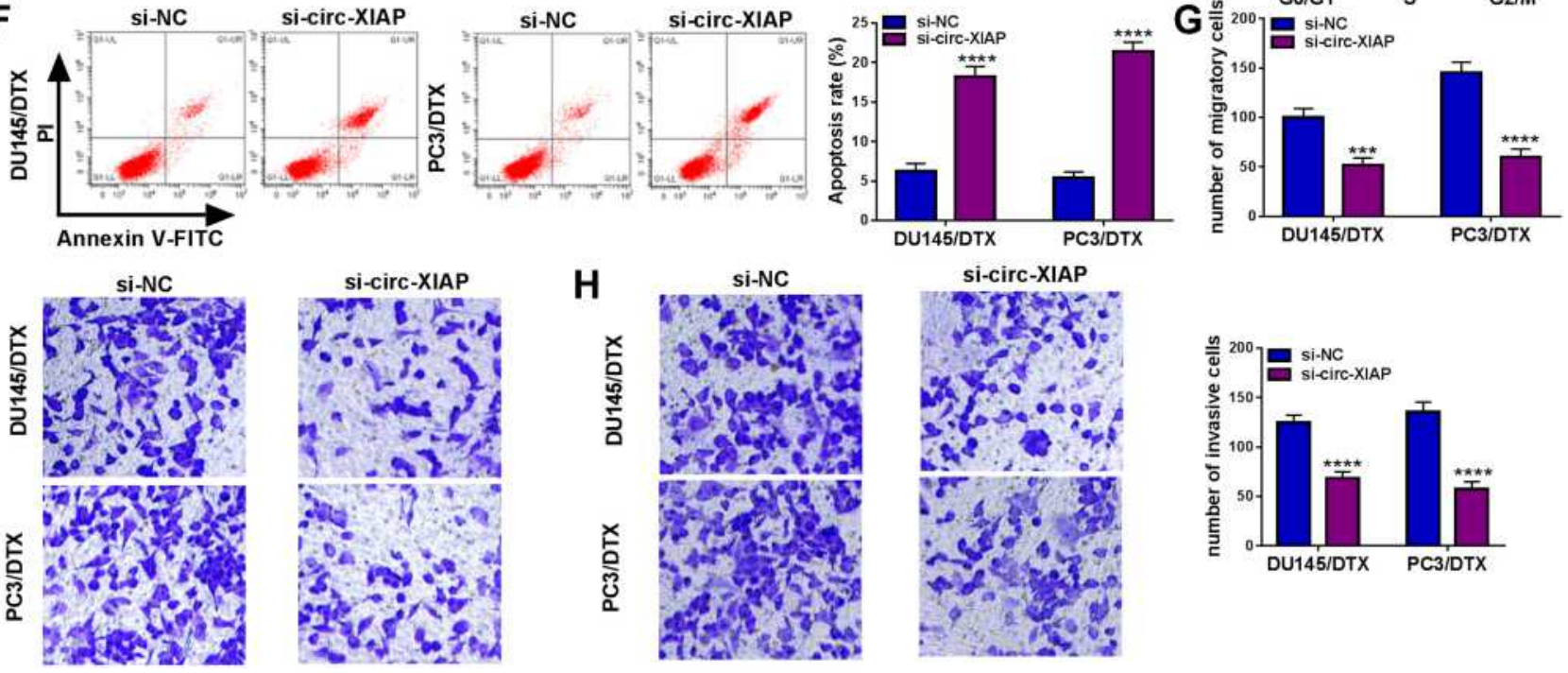

Figure 3 Circ-XIAP knockdown increased DTX sensitivity in DTX-resistant PCa cells. (A) Circ-XIAP expression was determined in DUI45/DTX and PC3/DTX cells after transfection with si-NC, si-circ-XIAP, vector, or circ-XIAP. (B-H) DUI45/DTX and PC3/DTX cells were introduced with si-NC or si-circ-XIAP. (B and C) IC 50 value of DTX and cell viability was examined via CCK-8 analysis. (D) The number of colonies was calculated using clone formation assay. (E and $F$ ) Flow cytometry was used to assess cell cycle distribution and cell apoptosis. (G and $\mathrm{H}$ ) Transwell assay was utilized to evaluate cell migration and invasion $(\times 100)$. $* * P<0.0 \mathrm{I}, * * * P<0.00 \mathrm{I}, * * * * P<0.000 \mathrm{I}$.

further study. As presented in Figure 4A, there existed potential binding sequence between circ-XIAP and miR-1182. To verify this prediction, dual-reporter luciferase assay was conducted. We observed that miR1182 upregulation obviously decreased the luciferase activity in WT-circ-XIAP group but not in MUT-circXIAP group (Figure 4B and C). Moreover, miR-1182 was found to be lowly expressed in resistant tissues compared with sensitive tissues (Figure 4D). Similarly,
miR-1182 expression in DU145 and PC3 cells was lower than RWPE-1 cells, and miR-1182 expression was the lowest in DU145/DTX and PC3/DTX cells (Figure 4E). A negative correlation between circXIAP and miR-1182 expression in resistant tissues was observed (Figure 4F). Furthermore, miR-1182 expression was increased when circ-XIAP was silenced in DTX-resistant cells, while miR-1182 expression was decreased after circ-XIAP overexpression (Figure 4G). 
A

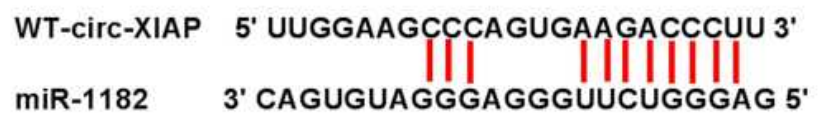

MUT-circ-XIAP 5' UUGGAAGGGGAGUGUUCUGGGAU 3'

B
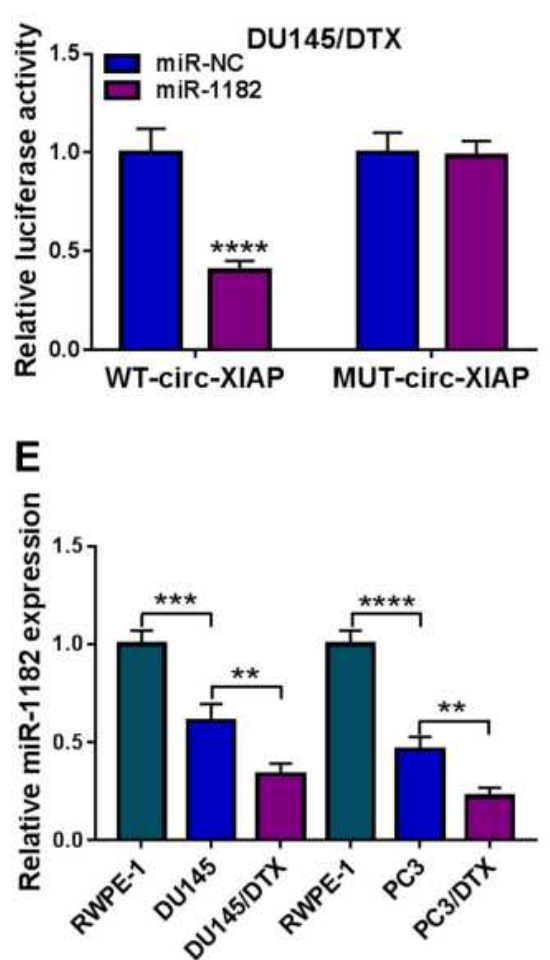

C

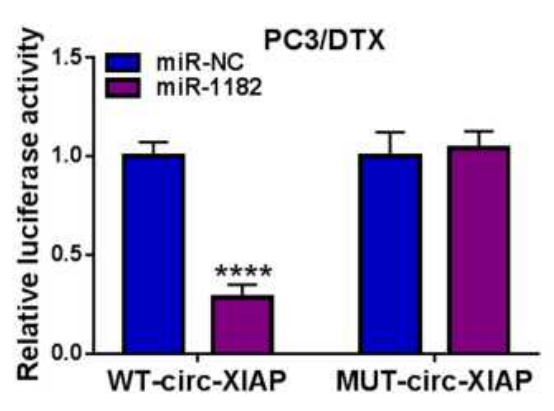

$\mathbf{F}$

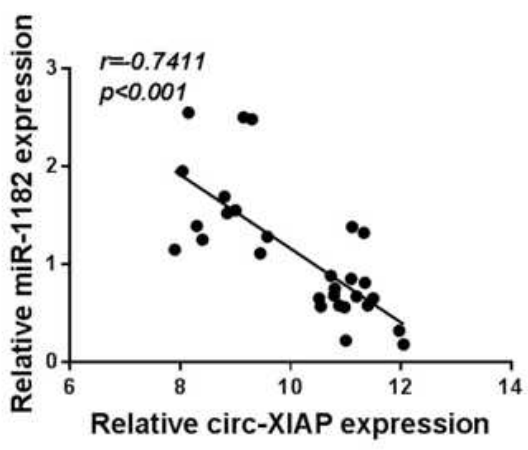

D
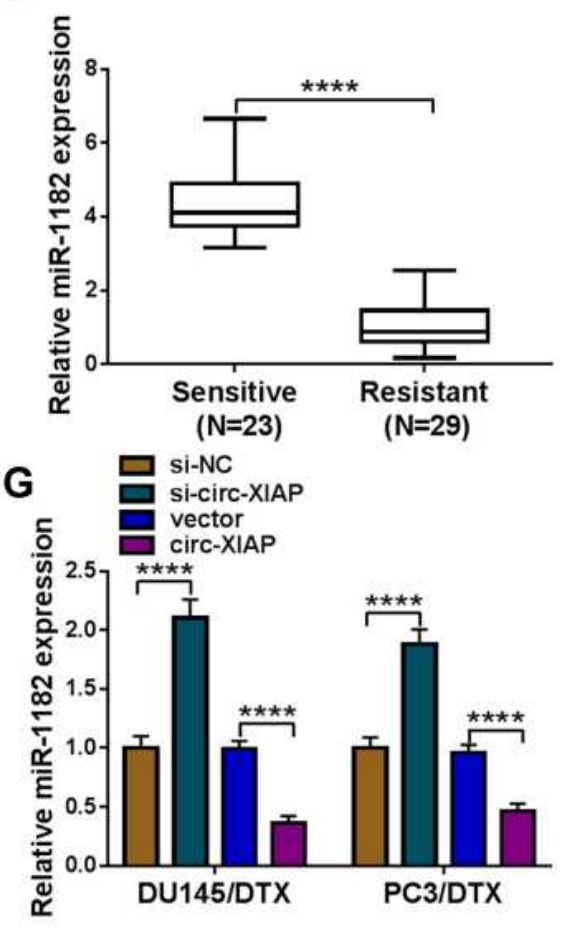

Figure 4 Circ-XIAP directly interacted with miR-II82. (A) The binding sequence between circ-XIAP and miR-II82 was shown. (B and C) The luciferase activity in DUI45/ DTX and PC3/DTX cells co-transfected with WT-circ-XIAP/MUT-circ-XIAP and miR-II82/miR-NC was detected. (D) MiR-II82 level in DTX-resistant tissues and DTXsensitive tissues was measured. (E) MiR-II 82 expression was analyzed in RWPE-I, DUI45, DUI45/DTX, PC3, and PC3/DTX cells. (F) The association between the levels of miR-I I82 and circ-XIAP in DTX-resistant tissues was analyzed. (G) The abundance of miR-I I82 was detected in DUI45/DTX and PC3/DTX cells after transfection with siNC, si-circ-XIAP, vector, or circ-XIAP. $* * P<0.01$, $* * * P<0.00$ I, $* * * * P<0.0001$.

These data concluded that circ-XIAP directly targeted miR-1182.

\section{Silencing Circ-XIAP Enhanced DTX Sensitivity in DTX-Resistant PCa Cells by Upregulating miR-I I82}

To determine whether the function of si-circ-XIAP was mediated by miR-1182, rescue experiments were performed in DU145/DTX and PC3/DTX. The expression of miR-1182 was increased by downregulating circ-XIAP, which was attenuated by inhibiting miR-1182 (Figure 5A). Moreover, we found that miR-1182 inhibition reversed the suppressive effects of circ-XIAP silence on cell viability and colony-forming ability (Figure 5B and C). Additionally, miR-1182 silence weakened si-circ-
XIAP-triggered cell cycle arrest and apoptosis (Figure 5D-F). Meanwhile, the inhibiting effects of circ-XIAP knockdown on migration and invasion were also counteracted by interference of miR-1182 (Figure 5G and H). Taken together, circ-XIAP regulated DTX sensitivity by targeting miR-1182.

\section{TPD52 Was a Direct Target of miR-I I82}

To explore the mechanisms by which miR-1182 regulated DTX resistance, TargetScan was employed to search for the candidate targets. There are many mRNAs might be targeted by miR-1182. We selected six common mRNAs involved in the development of PCa. We found that AKT3, YY1 and TPD52 expression levels were decreased after overexpression of miR-1182, and TPD52 expression was the lowest (Supplementary Figure 4). Therefore, TPD52 


\section{A}
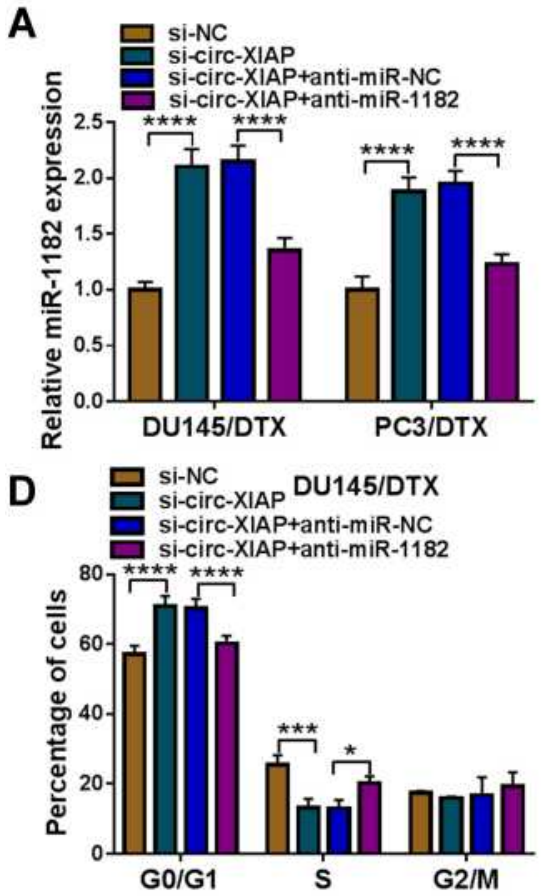

G

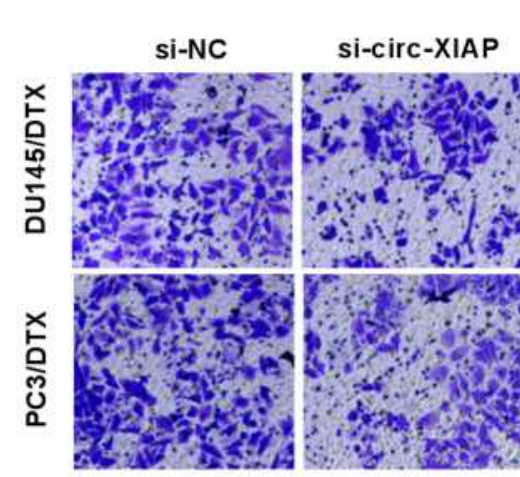

H
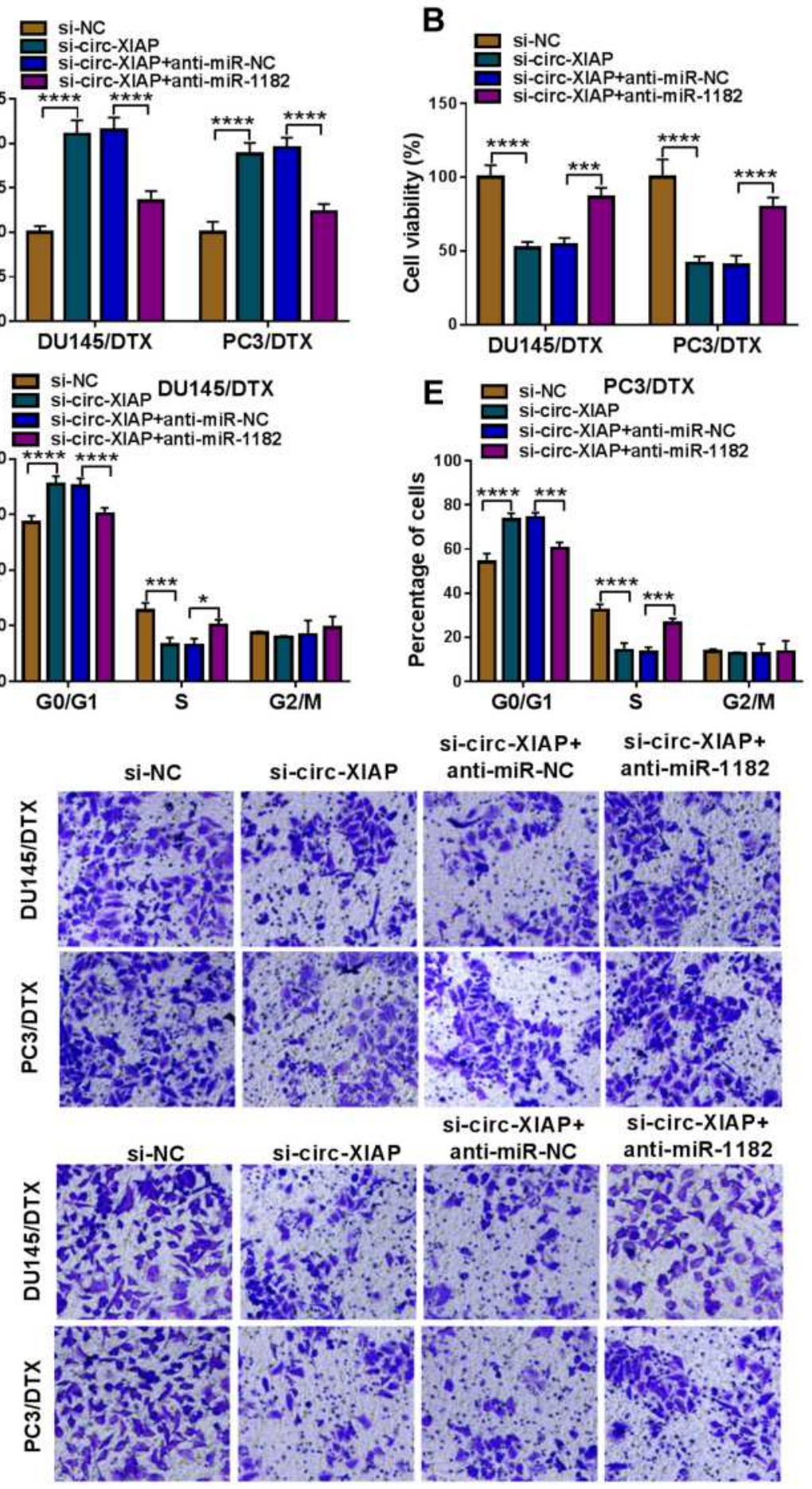

si-circ-XIAP+ si-circ-XIAP+ anti-miR-NC anti-miR-1182
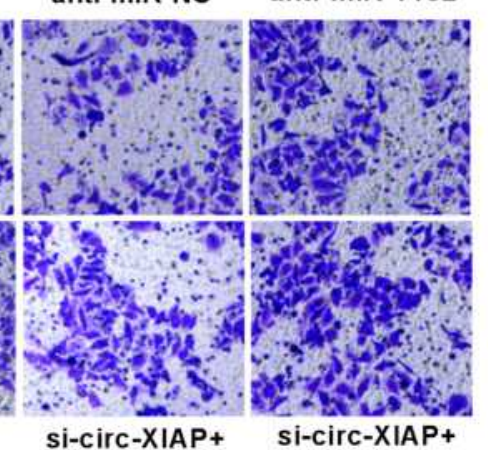
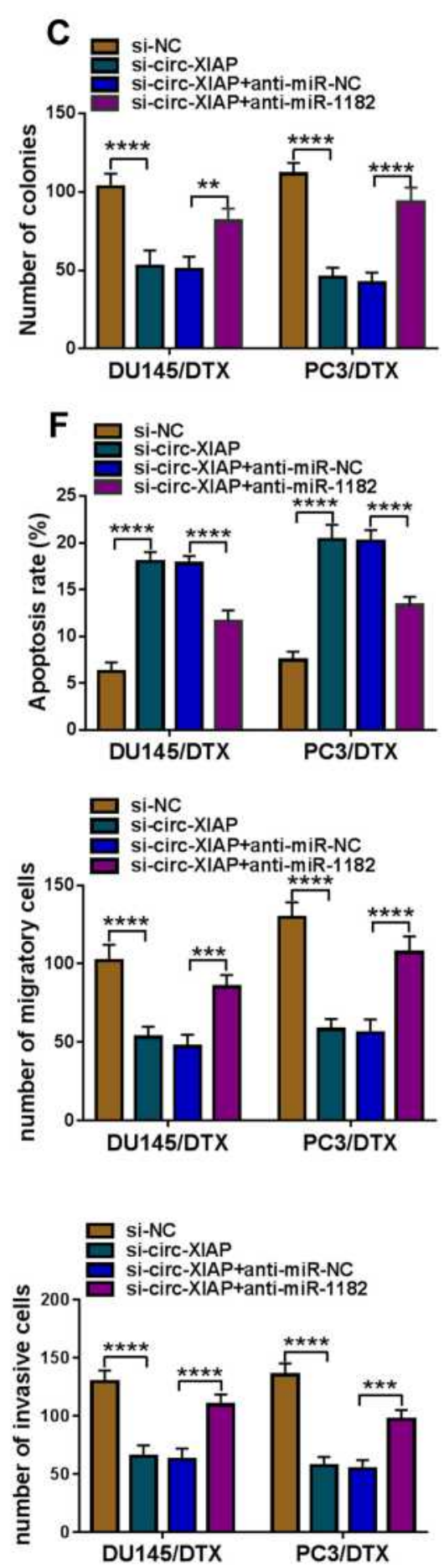

Figure 5 Downregulation of circ-XIAP improved sensitivity in DTX-resistant PCa cells by sponging miR-1/82. DUI45/DTX and PC3/DTX cells were transfected with siNC, si-circ-XIAP, si-circ-XIAP + anti-miR-NC, or si-circ-XIAP + anti-miR-I I82. (A) The expression of miR-II82 was tested. (B) CCK-8 assay was applied for analyzing cell viability. (C) Colony formation assay was used for evaluating colony-forming ability. (D-H) Cell cycle distribution, cell apoptosis, migration, and invasion were evaluated. $* P<0.05, * * P<0.01, * * * P<0.001, * * * * P<0.0001$.

was selected for further study. There were complementary sites between miR-1182 and TPD52 (Figure 6A), suggesting that miR-1182 might directly target TPD52. Moreover, miR-1182 overexpression evidently lowered the luciferase activity of WT-TPD52 3'UTR, while had no influence on the luciferase activity of MUT-TPD52 3'UTR (Figure 6B and C). We then investigated TPD52 expression in DTXresistant tissues and cells. TPD52 mRNA and protein 


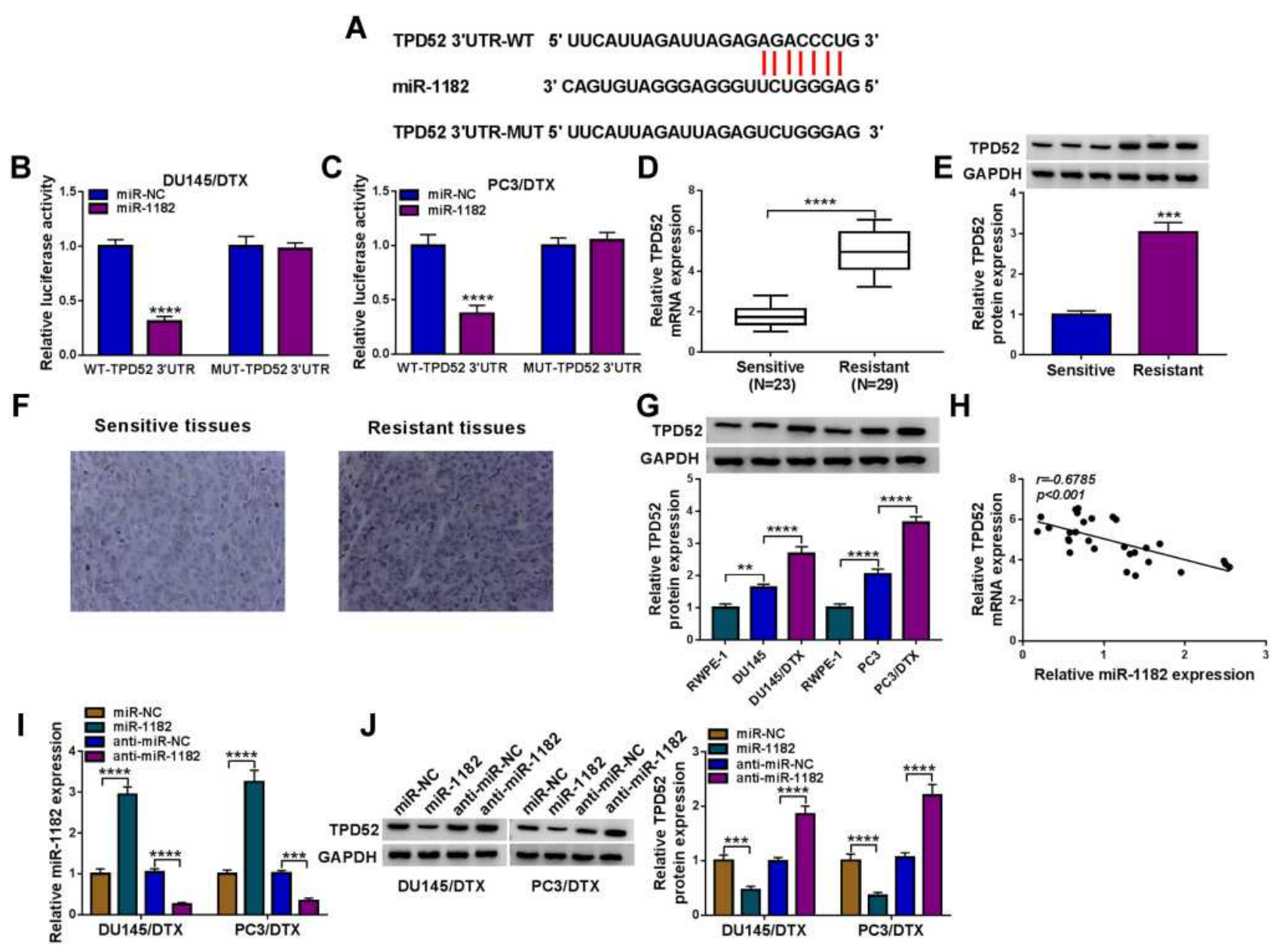

Figure 6 MiR-I I 82 directly targeted TPD52. (A) The binding sites between miR-I I82 and TPD52 were displayed. (B and C) The luciferase activity of DUI45/DTX and PC3/ DTX cells co-transfected with WT-TPD52 3'UTR/MUT-TPD52 3'UTR and miR-II82/miR-NC was assessed. (D and E) TPD52 mRNA and protein expression in DTXsensitive tissues and DTX-resistant tissues were measured. (F) Immunohistochemistry analysis was used to detect TPD52 positive cells in DTX-sensitive tissues and DTXresistant tissues. (G) TPD52 protein expression was measured in RWPE-I, DUI45, DUI45/DTX, PC3, and PC3/DTX cells. (H) The correlation between miR-II82 expression and TPD52 mRNA expression in DTX-resistant tissues was analyzed using. (I and J) MiR-I I 82 expression and TPD52 protein level in DUI45/DTX and PC3/DTX cells introduced with miR-NC, miR-II82, anti-miR-NC, or anti-miR-II82 were determined. $* * P<0.01, * * * P<0.001, * * * * P<0.0001$.

abundance were both higher in resistant tissues than sensitive tissues (Figure 6D and E). Immunohistochemistry analysis showed that TPD52 positive cells were increased in resistant tissues in comparison with sensitive tissues (Figure 6F). Likewise, TPD52 protein expression was found to be upregulated in DU145 and PC3 cells with respect to RWPE-1 cells, and TPD52 protein expression was further enhanced in DU145/DTX and PC3/DTX cells in contrast to parental cells (Figure 6G). Moreover, a distinct inverse correlation between miR-1182 expression and TPD52 mRNA in resistant tissues was revealed by Pearson correlation coefficient (Figure 6H). Inhibition and overexpression efficiency of miR-1182 were verified by qRT-PCR (Figure 6I). Additionally, we found that overexpression of miR-1182 inhibited the TPD52 protein level in DU145/DTX and PC3/DTX cells, and miR-1182 inhibition presented an opposite effect (Figure 6J). Therefore, we demonstrated that miR-1182 directly targeted TPD52 and negatively regulated its expression in DTX-resistant $\mathrm{PCa}$ cells.

\section{Overexpression of miR-I 182 Improved DTX Sensitivity in DTX-Resistant PCa Cells by Targeting TPD52}

To explore whether miR-1182 regulated DTX sensitivity by targeting TPD52, gain-of-function and rescue experiments were performed. WB data revealed that miR-1182 upregulation decreased the protein expression of TPD52, while TPD52 overexpression counteracted the inhibition effect of miR-1182 on TPD52 expression (Figure 7A). 


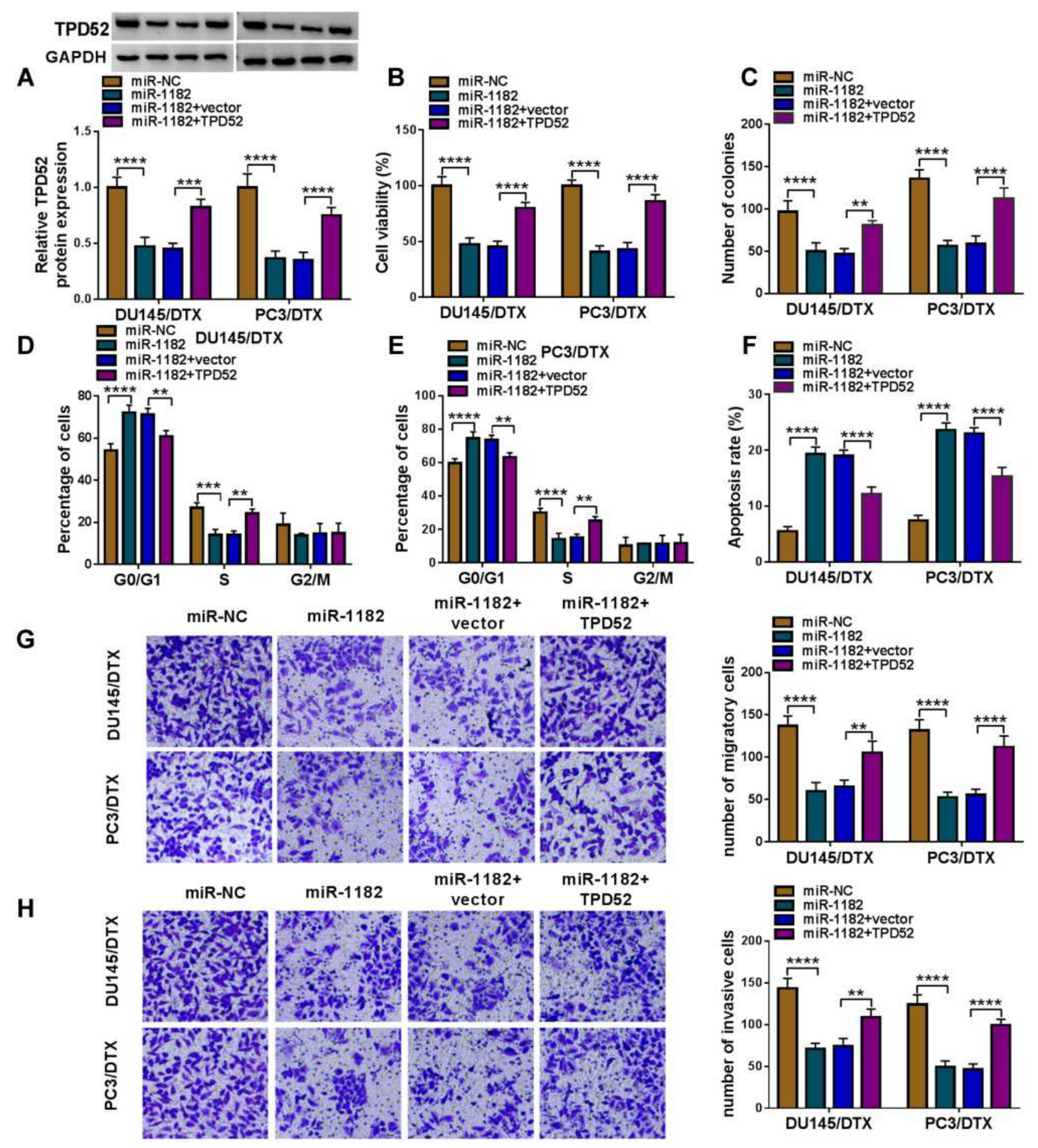

Figure 7 Upregulation of miR-I 82 enhanced DTX sensitivity in DTX-resistant PCa cells by downregulating TPD52. DUI45/DTX and PC3/DTX cells were transfected with miR-NC, miR-II82, miR-II82 + vector, or miR-II82 + TPD52. (A) The protein level of TPD52 was detected. (B and C) Cell viability and colony-forming ability were evaluated. (D-H) Cell cycle distribution, cell apoptosis, migration, and invasion were measured. $* * P<0.0 \mathrm{I}, * * * P<0.00 \mathrm{I}, * * * * P<0.000 \mathrm{I}$.

Moreover, we found that enforced expression of miR-1182 inhibited cell viability and colony-forming ability as well as induced cell cycle arrest and apoptosis in DU145/DTX and PC3/DTX cells, while these effects were reversed by addition of TPD52 (Figure 7B-F). Additionally, restoration of miR-1182 repressed migration and invasion of DU145/DTX and PC3/DTX cells, which could be abated by upregulating TPD52 (Figure $7 \mathrm{G}$ and H). These findings indicated that miR-1182 exerted its functions in DTX-resistant PCa cells by regulating TPD52. 


\section{Circ-XIAP Positively Modulated TPD52} Expression by Acting as a Sponge for miR-II 82

Next, we explored whether circ-XIAP could regulate TPD52 expression by sponging miR-1182. WB assay indicated that silence of circ-XIAP reduced TPD52 protein expression, whereas miR-1182 deficiency could neutralize this inhibition impact (Figure 8A and B), suggesting that circ-XIAP could regulate TPD52 expression by sponging miR-1182.

\section{Downregulation of Circ-XIAP Hampered Tumor Growth and Enhanced DTX}

\section{Sensitivity in vivo}

Transfection efficiency of sh-circ-XIAP was detected by transfection of sh-circ-XIAP (Figure 9A). To assess the role of circ-XIAP in tumor growth and DTX sensitivity in vivo, the mice xenograft model was established. Consistent with in vitro experiments, circ-XIAP silence or DTX treatment group showed smaller tumor volume and weight versus than that in the sh-NC group, and combination of circ-XIAP knockdown and DTX treatment further decreased tumor volume and weight compared to only DTX treatment

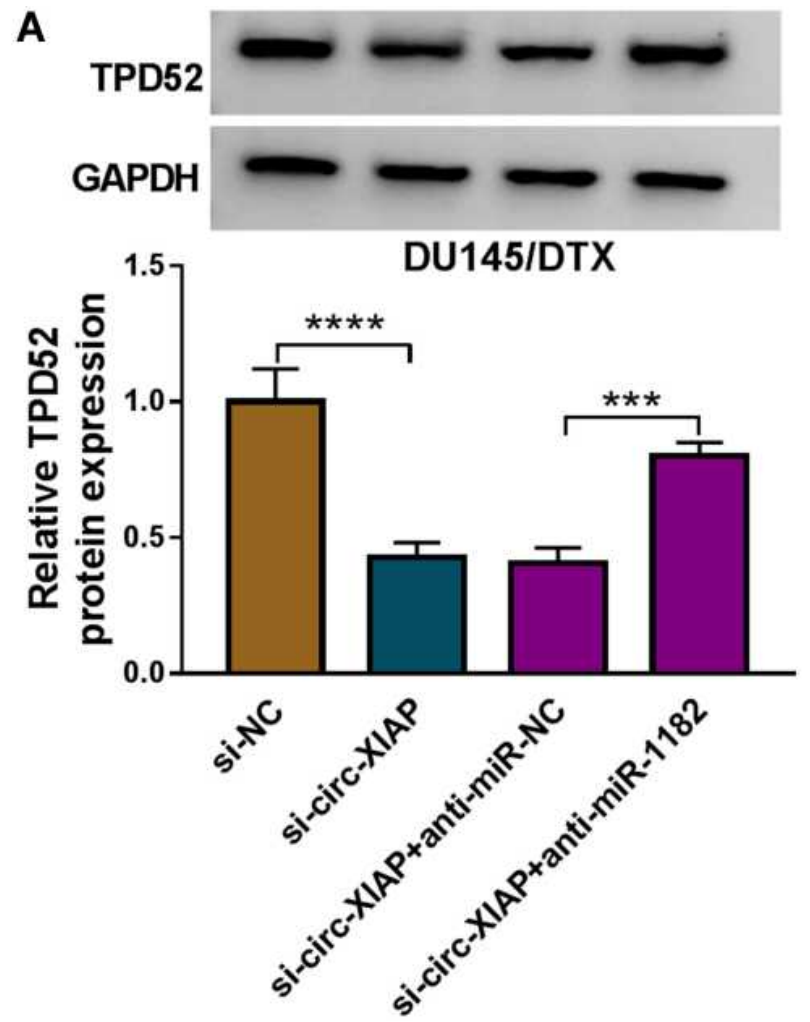

(Figure 9B and C). Moreover, circ-XIAP expression and TPD52 protein expression were significantly lower and miR-1182 expression was prominently higher in tumor tissues of sh-circ-XIAP group than that of sh-NC group (Figure 9D-F). The schematic diagram of the mechanism was presented in Figure 9G. Therefore, circ-XIAP silence suppressed tumor growth and promoted DTX sensitivity by upregulating miR-1182 and downregulating TPD52.

\section{Discussion}

$\mathrm{PCa}$ is frequent cancer that seriously threatens the health of men worldwide. ${ }^{28}$ Recently, the research on exosome function has attracted wide attention. It has been reported that exosome secretions, including circRNAs, not only regulate cancer progression but also affect chemosensitivity in human cancer. ${ }^{29,30}$ However, there are still few reports on the effect of exosomal circRNAs in DTX resistance of PCa. Therefore, the aim of our research was to probe the biological role of exosomal circ-XIAP in DTX resistance of PCa.

Recently, exosomes derived from tumor cells can stimulate tumor progression and serve as effective biomarkers for specific cancers. ${ }^{31,32}$ Moreover, the functions of exosomal circRNAs in cancer resistance have attracted

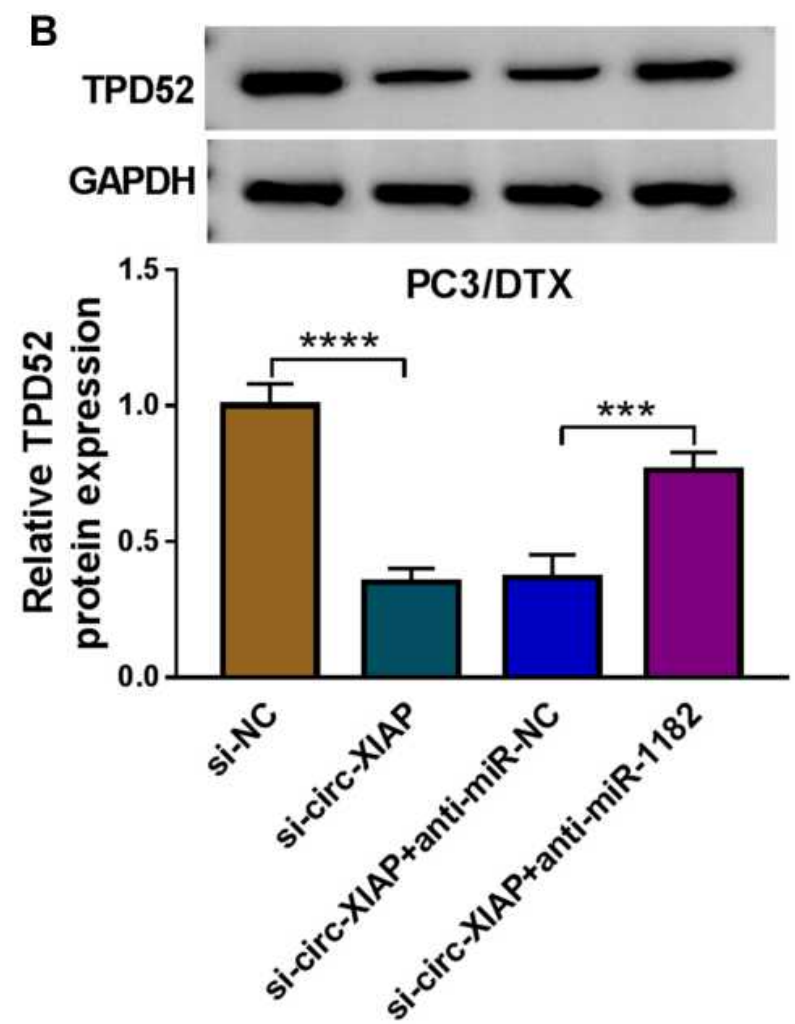

Figure 8 Circ-XIAP regulated TPD52 expression by sponging miR-I I82. (A and B) TPD52 protein expression was examined by WB in DUI45/DTX and PC3/DTX cells after introduction with si-NC, si-circ-XIAP, si-circ-XIAP + anti-miR-NC, or si-circ-XIAP + anti-miR-I I82. ***P<0.00I, *****P<0.000I. 
A

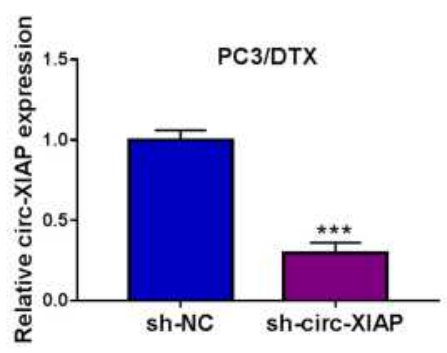

D

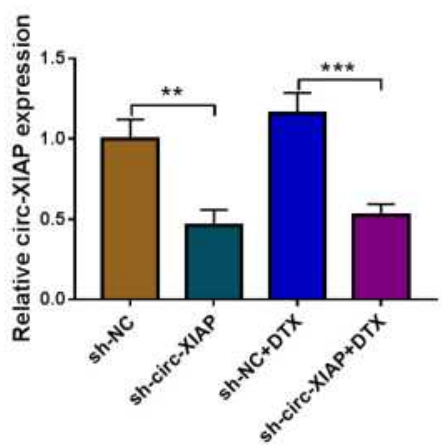

B

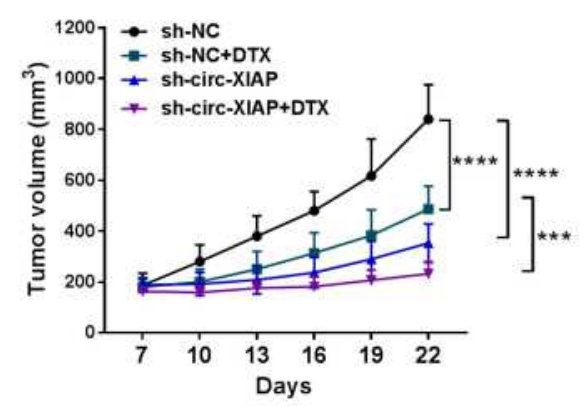

C
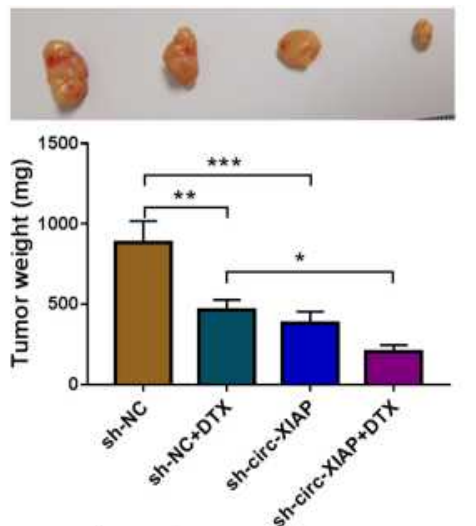

E

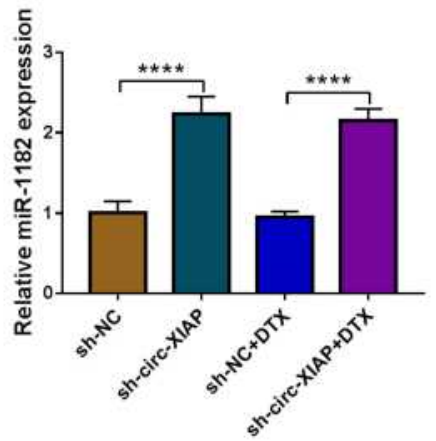

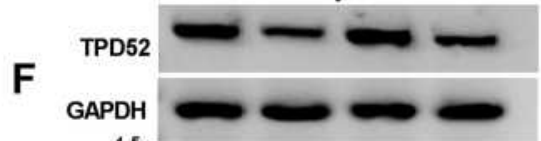

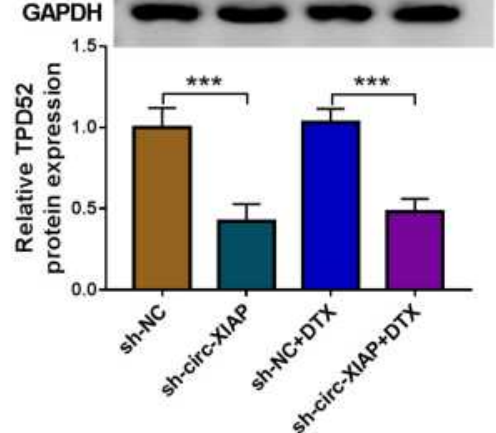

G

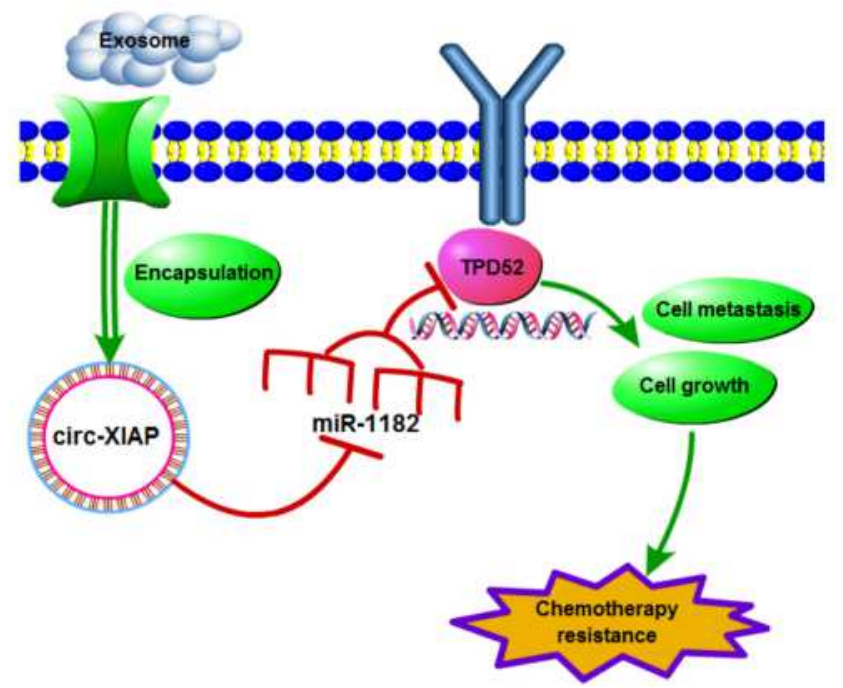

Figure 9 Silencing circ-XIAP promoted DTX sensitivity in vivo. (A) The expression of circ-XIAP in PC3/DTX cells transfected with sh-NC (as the control) or sh-circ-XIAP was measured by QRT-PCR. (B-F) PC3/DTX cells stably knocked down circ-XIAP were inoculated into nude mice, and the nude mice were treated with or without DTX $(10 \mathrm{mg} / \mathrm{kg}$ ) after injection for 7 days. (B) Tumor volume was monitored every 3 days. (C) After 22 days of injection, the weight of the tumor tissues was measured. (D and $E$ ) Circ-XIAP and miR-II82 levels in tumor tissues of nude mice were determined. (F) TPD52 protein level was analyzed in tumor tissues. (G) Schematic diagram of the mechanism in which exosomal circ-XIAP modulated chemotherapy resistance by regulating miR-II $82 / T P D 52$ axis. $* P<0.05, * * P<0.01, * * * P<0.00 \mathrm{I}, * * * * P<0.000 \mathrm{I}$.

increasing interest. For example, Han et al revealed that exosomal circ-HIPK3 facilitated temozolomide resistance and tumor progression via modulating miR-421/ZIC5 axis in glioma. ${ }^{33}$ Moreover, Hu et al found that exosomederived circ_UBE2D2 promoted resistance of breast cancer to tamoxifen via regulating miR-200a-3p. ${ }^{34}$ Circ-XIAP has been found to be upregulated and promoted proliferation and migration in $\mathrm{PCa}^{20}$ However, the roles of exosomal circ-XIAP in DTX resistance of PCa have not been reported. In our research, circ-XIAP expression was increased in DTX-resistant PCa cells and tissues. Next, cell exosomes were extracted from DTX-resistant PCa 
cells, and the vesicles were shown to be round shape with double membranes, and the diameter was from $40 \mathrm{~nm}$ to $100 \mathrm{~nm}$ under a TEM. Subsequently, we observed that circ-XIAP was significantly enriched in exosomes from DTX-resistant PCa cells and could be transferred to parental cells after treatment with exosomes. These data indicated that circ-XIAP could be transferred via exosomes. Moreover, circ-XIAP deletion mitigated DTX resistance by suppressing resistant cell growth, migration and invasion and inducing cell cycle arrest and cell apoptosis, indicating the involvement of circ-XIAP in the regulating DTX resistance.

To study the mechanism by which circ-XIAP functions in DTX resistance, we confirmed its intracellular localization, and found that circ-XIAP was predominantly located in the cytoplasm. Given that circRNAs have been widely studied as sponges for miRNAs and circ-XIAP had a high abundance in the cytoplasm, we next explored whether circXIAP acted as a sponge for miRNA. As we expected, miR1182 was identified as a target of circ-XIAP. MiRNAs were reported to be involved in the pathological processes of diverse diseases through regulating cell growth, cell cycle, metastasis, apoptosis, and drug resistance. ${ }^{3,36}$ MiR-1182 has been demonstrated to suppress cell tumorigenesis in diverse cancers, such as ovarian cancer, ${ }^{37}$ gastric cancer, ${ }^{38}$ bladder cancer ${ }^{39}$ and so on. Moreover, Huang et al demonstrated that miR-1182 was lowly expressed in PCa tissue samples, and miR-1182 deficiency abolished the repressive impact of circABCC4 silence on PCa progression. ${ }^{24}$ Our study displayed that miR-1182 abundance was decreased in DTX-resistant PCa tissue samples and cells. Subsequent experiments showed that miR-1182 downregulation could abate the effects of si-circ-XIAP on DTX-resistant cell growth, cell cycle, apoptosis, migration, and invasion. In a word, our results implied that circ-XIAP knockdown enhanced DTX sensitivity by sponging miR-1182 in DTXresistant PCa cells.

To explore how miR-1182 regulated the DTX resistance of $\mathrm{PCa}$, possible targets of miR-1182 were predicted using the online tool (TargetScan). We demonstrated that TPD52 was targeted by miR-1182. TPD52, a tumor-promoting gene, is usually upregulated in many tumors, including PCa. ${ }^{40,41}$ More importantly, TPD52 also was reported to increase DTX resistance of PCa via suppressing LKB1/ AMPK-mediated autophagy. ${ }^{42}$ Here, we uncovered that TPD52 level was elevated in DTX-resistant PCa tissue samples and cells. Moreover, TPD52 elevation could counteract the promotive effect of miR-1182 on DTX sensitivity.
In addition, circ-XIAP inhibition could directly decrease TPD52 expression via sponging miR-1182. Meanwhile, circ-XIAP depletion restrained DTX-resistant tumor growth and improved DTX sensitivity via regulation of miR-1182 and TPD52 expression. These results suggested that circXIAP contributed to the development of DTX-resistant PCa cells by regulating miR-1182/TPD52 axis.

In conclusion, we revealed that exosome-mediated circ-XIAP could enhance DTX resistance of PCa by regulating miR-1182/TPD52 axis. Our study offered a novel insight into the roles of exosomes in PCa and offered a promising therapeutic target to improve chemotherapy efficiency for PCa patients.

\section{Funding}

There is no funding to report.

\section{Disclosure}

The authors declare that they have no conflicts of interest.

\section{References}

1. Bray F, Ferlay J, Soerjomataram I, et al. Global cancer statistics 2018: GLOBOCAN estimates of incidence and mortality worldwide for 36 cancers in 185 countries. CA Cancer J Clin. 2018;68 (6):394-424. doi:10.3322/caac.21492

2. Wu J, Cheng G, Zhang C, et al. Long noncoding RNA LINC01296 is associated with poor prognosis in prostate cancer and promotes cancer-cell proliferation and metastasis. Onco Targets Ther. 2017;10:1843-1852. doi:10.2147/OTT.S129928

3. Galsky MD, Vogelzang NJ. Docetaxel-based combination therapy for castration-resistant prostate cancer. Ann Oncol. 2010;21 (11):2135-2144. doi:10.1093/annonc/mdq050

4. Sekino Y, Oue N, Koike Y, et al. KIFC1 inhibitor CW069 induces apoptosis and reverses resistance to docetaxel in prostate cancer. J Clin Med. 2019;8(2):225. doi:10.3390/jcm8020225

5. Cocucci E, Meldolesi J. Ectosomes and exosomes: shedding the confusion between extracellular vesicles. Trends Cell Biol. 2015;25 (6):364-372. doi:10.1016/j.tcb.2015.01.004

6. Bei Y, Das S, Rodosthenous RS, et al. Extracellular vesicles in cardiovascular theranostics. Theranostics. 2017;7(17):4168-4182. doi: $10.7150 /$ thno.21274

7. Takahashi K, Yan IK, Kogure T, et al. Extracellular vesicle-mediated transfer of long non-coding RNA ROR modulates chemosensitivity in human hepatocellular cancer. FEBS Open Bio. 2014;4(1):458-467. doi:10.1016/j.fob.2014.04.007

8. Vlassov AV, Magdaleno S, Setterquist R, et al. Exosomes: current knowledge of their composition, biological functions, and diagnostic and therapeutic potentials. Biochim Biophys Acta. 2012;1820 (7):940-948. doi:10.1016/j.bbagen.2012.03.017

9. Ge R, Tan E, Sharghi-Namini S, et al. Exosomes in cancer microenvironment and beyond: have we overlooked these extracellular messengers? Cancer Microenviron. 2012;5(3):323-332. doi:10.1007/s12307-012-0110-2

10. Cui J, Wang H, Zhang X, et al. Exosomal miR-200c suppresses chemoresistance of docetaxel in tongue squamous cell carcinoma by suppressing TUBB3 and PPP2R1B. Aging (Albany NY). 2020;12 (8):6756-6773. doi:10.18632/aging. 103036 
11. Malla B, Zaugg K, Vassella E, et al. Exosomes and exosomal microRNAs in prostate cancer radiation therapy. Int J Radiat Oncol Biol Phys. 2017;98(5):982-995. doi:10.1016/j.ijrobp.2017.03.031

12. Memczak S, Jens M, Elefsinioti A, et al. Circular RNAs are a large class of animal RNAs with regulatory potency. Nature. 2013;495 (7441):333. doi:10.1038/nature11928

13. Chen LL. The biogenesis and emerging roles of circular RNAs. Nat Rev Mol Cell Biol. 2016;17(4):205-211. doi:10.1038/nrm.2015.32

14. Arnaiz E, Sole C, Manterola L, et al. CircRNAs and cancer: biomarkers and master regulators. Semin Cancer Biol. 2019;58:90-99. doi:10.1016/j.semcancer.2018.12.002

15. Vo JN, Cieslik M, Zhang Y, et al. The landscape of circular RNA in cancer. Cell. 2019;176(4):869-881.e813. doi:10.1016/j.cell.2018.12.021

16. Hong X, Liu N, Liang Y, et al. Circular RNA CRIM1 functions as a ceRNA to promote nasopharyngeal carcinoma metastasis and docetaxel chemoresistance through upregulating FOXQ1. Mol Cancer. 2020;19(1):33. doi:10.1186/s12943-020-01149-x

17. Gao Y, Liu J, Huan J, et al. Downregulation of circular RNA hsa_circ_0000735 boosts prostate cancer sensitivity to docetaxel via sponging miR-7. Cancer Cell Int. 2020;20(1):334. doi:10.1186/ s12935-020-01421-6

18. Li Y, Zheng Q, Bao C, et al. Circular RNA is enriched and stable in exosomes: a promising biomarker for cancer diagnosis. Cell Res. 2015;25(8):981-984. doi:10.1038/cr.2015.82

19. Wang Y, Liu J, Ma J, et al. Exosomal circRNAs: biogenesis, effect and application in human diseases. Mol Cancer. 2019;18(1):116. doi:10.1186/s12943-019-1041-Z

20. Feng Y, Yang Y, Zhao X, et al. Circular RNA circ0005276 promotes the proliferation and migration of prostate cancer cells by interacting with FUS to transcriptionally activate XIAP. Cell Death Dis. 2019;10 (11):792. doi:10.1038/s41419-019-2028-9

21. Bach DH, Lee SK, Sood AK. Circular RNAs in Cancer. Mol Ther Nucleic Acids. 2019;16:118-129. doi:10.1016/j.omtn.2019.02.005

22. Hou XS, Han CQ, Zhang W. MiR-1182 inhibited metastasis and proliferation of ovarian cancer by targeting hTERT. Eur Rev Med Pharmacol Sci. 2018;22(6):1622-1628. doi:10.26355/eurrev_ 20180314569

23. Xiao J, Yu H, Ma Z. LINC00339 promotes growth and invasiveness of hepatocellular carcinoma by the miR-1182/SKA1 pathway. Onco Targets Ther. 2019;12:4481-4488. doi:10.2147/OTT.S207397

24. Huang C, Deng H, Wang Y, et al. Circular RNA circABCC4 as the ceRNA of miR-1182 facilitates prostate cancer progression by promoting FOXP4 expression. J Cell Mol Med. 2019;23(9):6112-6119. doi:10.1111/jcmm. 14477

25. Byrne JA, Balleine RL, Schoenberg Fejzo M, et al. Tumor protein D52 (TPD52) is overexpressed and a gene amplification target in ovarian cancer. Int J Cancer. 2005;117(6):1049-1054. doi:10.1002/ijc.21250

26. Dasari C, Yaghnam DP, Walther R, et al. Tumor protein D52 (isoform 3) contributes to prostate cancer cell growth via targeting nuclear factor $-\kappa \mathrm{B}$ transactivation in LNCaP cells. Tumour Biol. 2017;39(5):1010428317698382. doi:10.1177/1010428317698382

27. Su Q, Lv X. Revealing new landscape of cardiovascular disease through circular RNA-miRNA-mRNA axis. Genomics. 2020;112 (2):1680-1685. doi:10.1016/j.ygeno.2019.10.006
28. Schatten H. Brief overview of prostate cancer statistics, grading, diagnosis and treatment strategies. Adv Exp Med Biol. 2018;1095:1-14.

29. Shi X, Wang B, Feng X, et al. circRNAs and exosomes: a mysterious frontier for human cancer. Mol Ther Nucleic Acids. 2020;19:384-392. doi:10.1016/j.omtn.2019.11.023

30. Wang $\mathrm{X}$, Zhang $\mathrm{H}$, Yang $\mathrm{H}$, et al. Exosome-delivered circRNA promotes glycolysis to induce chemoresistance through the miR-122PKM2 axis in colorectal cancer. Mol Oncol. 2020;14(3):539-555. doi:10.1002/1878-0261.12629

31. Duijvesz D, Luider T, Bangma $\mathrm{CH}$, et al. Exosomes as biomarker treasure chests for prostate cancer. Eur Urol. 2011;59(5):823-831. doi:10.1016/j.eururo.2010.12.031

32. Li W, Li C, Zhou $\mathrm{T}$, et al. Role of exosomal proteins in cancer diagnosis. Mol Cancer. 2017;16(1):145. doi:10.1186/s12943-0170706-8

33. Han C, Wang S, Wang H, et al. Exosomal circ-HIPK3 facilitates tumor progression and temozolomide resistance by regulating miR-421/ZIC5 axis in glioma. Cancer Biother Radiopharm. 2020.

34. $\mathrm{Hu} \mathrm{K}$, Liu X, Li Y, et al. Exosomes mediated transfer of circ_UBE2D2 enhances the resistance of breast cancer to tamoxifen by binding to miR-200a-3p. Med Sci Monit. 2020;26:e922253. doi:10.12659/MSM.922253

35. Tutar Y. miRNA and cancer; computational and experimental approaches. Curr Pharm Biotechnol. 2014;15(5):429. doi:10.2174/ 138920101505140828161335

36. Mishra S, Yadav T, Rani V. Exploring miRNA based approaches in cancer diagnostics and therapeutics. Crit Rev Oncol Hematol. 2016;98:12-23. doi:10.1016/j.critrevonc.2015.10.003

37. Zong ZH, Du YP, Guan X, et al. CircWHSC1 promotes ovarian cancer progression by regulating MUC1 and hTERT through sponging miR-145 and miR-1182. J Exp Clin Cancer Res. 2019;38(1):437. doi:10.1186/s13046-019-1437-z

38. Zhang D, Xiao YF, Zhang JW, et al. miR-1182 attenuates gastric cancer proliferation and metastasis by targeting the open reading frame of hTERT. Cancer Lett. 2015;360(2):151-159. doi:10.1016/j. canlet.2015.01.044

39. Zhou J, Dai W, Song J. miR-1182 inhibits growth and mediates the chemosensitivity of bladder cancer by targeting hTERT. Biochem Biophys Res Commun. 2016;470(2):445-452. doi:10.1016/j. bbrc.2016.01.014

40. Li J, Li Y, Liu H, et al. The four-transmembrane protein MAL2 and tumor protein D52 (TPD52) are highly expressed in colorectal cancer and correlated with poor prognosis. PLoS One. 2017;12(5):e0178515. doi:10.1371/journal.pone.0178515

41. Ummanni R, Teller S, Junker H, et al. Altered expression of tumor protein D52 regulates apoptosis and migration of prostate cancer cells. Febs j. 2008;275(22):5703-5713. doi:10.1111/j.17424658.2008.06697.x

42. Zeng J, Liu W, Fan YZ, et al. PrLZ increases prostate cancer docetaxel resistance by inhibiting LKB1/AMPK-mediated autophagy. Theranostics. 2018;8(1):109-123. doi:10.7150/thno.20356

\section{Publish your work in this journal}

Drug Design, Development and Therapy is an international, peerreviewed open-access journal that spans the spectrum of drug design and development through to clinical applications. Clinical outcomes, patient safety, and programs for the development and effective, safe, and sustained use of medicines are a feature of the journal, which has also been accepted for indexing on PubMed Central. The manuscript management system is completely online and includes a very quick and fair peer-review system, which is all easy to use. Visit http://www. dovepress.com/testimonials.php to read real quotes from published authors. 